\title{
Comportamiento del consumidor y turismo sostenible*
}

\author{
Jorge E. Araña \\ Carmelo J. León \\ Universidad de Las Palmas de Gran Canaria
}

\section{Resumen}

El turismo es una industria que ha demostrado un alto potencial para generar bienestar humano en muchos países y regiones del planeta. Sin embargo, este potencial está condicionado por el objetivo de la sostenibilidad, que implica la compatibilización de los equilibrios ambientales, sociales y económicos. Para conseguir el objetivo de la sostenibilidad es necesario modificar los comportamientos de los turistas como consumidores. En este trabajo se presentan las ideas centrales sobre las que giran las decisiones de los turistas en el uso de los recursos naturales y el impacto sobre el medio ambiente. Se constata que el turismo, como industria hedonística, no presenta suficientes incentivos a los turistas para realizar un comportamiento ambientalmente responsable. Por ello, se necesita actuar desde una concepción de un modelo de decisión turística integrador, en el que se combinen los instrumentos económicos con los condicionantes del contexto en la toma de decisiones de los turistas. Se presentan resultados de dos experimentos relacionados con las decisiones sobre emisiones de $\mathrm{CO}_{2}$ que afectan al cambio climático y con las decisiones de reutilización de enseres en los establecimientos alojativos. La evidencia aportada permite concluir que los instrumentos de política ambiental pueden complementarse con los instrumentos que afecten al contexto en la toma de decisiones, como la información y las normas sociales, para modificar los comportamientos de los turistas hacia decisiones social y ambientalmente más responsables.

Palabras clave: comportamiento del consumidor, brecha de las actitudes y acciones, emociones, información, turismo sostenible.

Clasificación JEL: L83, H23, Z32.

\begin{abstract}
Tourism is an industry that has demonstrated a high potential to generate human wellbeing in many countries and regions of the planet. However, this potential is conditioned by the objective of sustainability, which implies the compatibility of environmental, social and economic aspects. To achieve the goal of sustainability it is necessary to modify the behaviour of tourists as consumers. This paper presents the central ideas revolving around the decisions of tourists in the use of natural resources and the impact on the environment. It is noted that tourism, as a hedonistic industry, does not present enough incentives for tourists to perform environmentally responsible behaviour. For this reason, it is necessary to act from an integrated model of tourist decision, in which economic instruments are combined with the contextual factors conditioning the decision making of tourists. Results are presented on two experiments related to decisions on $\mathrm{CO}_{2}$ emissions that affect climate change and decisions on the reuse towels and linen in accommodation establishments. The evidence provided allows us to conclude that environmental
\end{abstract}

* Los autores desean agradecer la financiación del Ministerio de Economía y Competitividad, a través de Fondos FEDER de la Unión Europea, mediante el Proyecto ECO2014-60058-P. 
policy instruments can be complemented with instruments that affect the context in decisionmaking, such as information and social norms, in order to modify the behaviour of tourists towards socially and environmentally more responsible decisions.

Keywords: consumer behaviour, gap of attitudes and actions, emotions, information, sustainable tourism.

JEL classification: L83, H23, Z32.

\section{Introducción}

Los turistas toman decisiones de consumo en diferentes puntos de un proceso que va desde la expectativa sobre la realización de un viaje, la contratación del mismo, el encuentro con el destino, y el recordatorio de la experiencia vivida (Gnoth, 1997; Aho, 2001; Gretzel et al., 2006). En todas estas fases del proceso de consumo turístico se pueden observar impactos ambientales de diverso grado, ocasionados por las decisiones de elección de los turistas. El camino hacia una economía turística sostenible conlleva la formulación de políticas que induzcan a los turistas a tomar las decisiones ambientalmente más sostenibles en cada etapa del proceso turístico, de modo que se logre una minimización de impactos ambientales consistente con un equilibrio con los parámetros ambientales.

No cabe duda que los turistas pretenden el objetivo de la satisfacción y la felicidad obtenida con las decisiones involucradas en el viaje, pero este objetivo individual debe ser compatible con el objetivo global de la sostenibilidad (Becken, 2007; Cohen y Higham, 2011; Gössling et al., 2012; Gössling, 2015). Por ejemplo, el turismo contribuye con el 5 por 100 de las emisiones globales de $\mathrm{CO}_{2}$, y se estima que entre el 5,2 y el 12,5 por 100 de las emisiones antropogénicas de $\mathrm{CO}_{2}$ son debidas al turismo (Gössling, 2015; Scott et al., 2016). Se trata, por tanto, de una industria que tiene aún un largo camino para reducir el impacto que está teniendo al cambio climático, y esto solo puede lograrse a partir del cambio de modelos de consumo y procesos de decisión.

El diseño de políticas de sostenibilidad ambiental debe tener en cuenta las posibles reacciones de los turistas. La utilización de modelos de comportamiento racional puede conducir a estimaciones sesgadas cuando se comparan con el comportamiento real en los mercados turísticos. A su vez, los mecanismos de intervención basados en el uso de incentivos, como los impuestos ambientales (Sheng y Tsui, 2009; Palmer y Riera, 2003; Gooroochurn y Milner, 2005; Gössling et al., 2007), pueden no ser tan eficaces cuando intervienen otros aspectos que influyen en el comportamiento de los turistas, como las emociones o las normas sociales (Gnoth, 1997; King, 2002; Gretzel et al., 2006).

Por ello, teniendo en cuenta la naturaleza racional y emocional en la que se desenvuelve el contexto de la toma de decisiones de los consumidores, se necesita la consideración de un modelo más completo de los factores que influyen en los resultados finales de las elecciones turísticas (Lerner y Keltner, 2000; Barsky y Nash, 2002; Antimova, Nawijn y Peeters, 2012). Tanto las emociones, como los factores que 
influyen en la capacidad cognitiva de los agentes al tomar sus decisiones, pueden condicionar los comportamientos sostenibles de los turistas, explicando las divergencias observadas entre las intenciones o actitudes -racionales- y los comportamientos observados que generan un mayor impacto ambiental que el esperado (Hibbert et al., 2013; Hassan et al., 2016).

Por ejemplo, los turistas pueden tener una alta conciencia ambiental en el ahorro de agua y desarrollar comportamientos ahorradores en sus residencias habituales, pero comportarse como consumidores más desperdiciadores en los lugares de destino (Miller et al., 2015). Esta divergencia entre el comportamiento en origen y en destino en cuanto a los aspectos ambientales, ha sido sugerida por los resultados encontrados en Juvan et al. (2016), Higham et al. (2016) y Doran et al. (2017), entre otros. En cierto modo, el desfase entre las actitudes y los comportamientos observados tiende a potenciarse en contextos hedonísticos como el turístico, en los que prima la satisfacción y el placer fuera de la residencia habitual, como mecanismo de recuperación física y mental lejos de las actividades cotidianas. En este contexto, el turista tiene la tentación de permitirse comportamientos ambientalmente despilfarradores que no llevaría a cabo en su lugar de origen (Higham y Cohen, 2011). La implementación de políticas que corrijan este tipo de desfase comportamental es uno de los retos más importantes que presenta el objetivo de la sostenibilidad turística.

Los comportamientos turísticos poco sostenibles son evidentes en la utilización del agua como recurso. La escasez de agua en entornos turísticos locales es uno de los impactos más relevantes que se avecinan por el cambio climático a nivel global (Vörösmarty et al., 2000; Scott et al., 2012; Gosling y Arnell, 2016). Además, el agua es un recurso muy utilizado en la industria turística, para el que la utilización eficiente puede conducir a la reducción de costes económicos y energéticos. Los esfuerzos en la reducción de agua en el turismo han sido dirigidos a la utilización eficiente en la producción de los servicios de alimentación, irrigación, y habitaciones. Por ejemplo, estos esfuerzos han llevado a muchos hoteles y establecimientos alojativos a implementar medidas para convencer a los turistas de la conveniencia de la reutilización de las toallas y la ropa de cama en las habitaciones (Baca Motes et al., 2013; Dolnicar et al., 2016; Goldstein et al., 2008, 2011; Mair y Bergin-Seers 2010; Shang et al., 2010). Muchos hoteles cambian estos enseres una vez cada día durante la estancia del turista. Sin duda, la reducción de la utilización de las toallas y ropa de cama al menos durante un día adicional puede reducir los costes de lavandería y energía incurridos en la gestión de los enseres a la mitad. Se trata de reducciones importantes de costes que suponen unos menores impactos ambientales y un comportamiento más sostenible por parte de la industria turística, sin que suponga una reducción relevante de la calidad del servicio.

En este trabajo se analizan los determinantes del comportamiento sostenible de los turistas en aspectos relacionados con decisiones que contribuyen a las emisiones de $\mathrm{CO}_{2}$ y al consumo de agua durante la experiencia turística. Se presentan resultados de dos experimentos relacionados con las decisiones de elección de emisiones de $\mathrm{CO}_{2}$ y de consumo de agua y energía. El primer experimento se centra en la elección 
de paquetes turísticos con distintos niveles de emisiones de $\mathrm{CO}_{2}$, mientras que el segundo analiza las decisiones de reutilización de toallas y ropa de cama en los establecimientos alojativos. Los ejemplos mostrados en este trabajo revelan que es posible influir en los comportamientos de los turistas hacia la adopción de decisiones de elección más sostenibles, actuando sobre las variables que influyen en el contexto de la elección, como la información, el estado emocional y las normas sociales.

\section{Comportamiento ambiental y sostenibilidad turística}

La sostenibilidad de la industria turística involucra una gestión equilibrada entre los aspectos ambientales, sociales y económicos (Butler, 1999; Sharpley, 2000; Jamal, 2014). El turismo es una industria que genera un amplio bienestar económico para las sociedades de destino, pero al mismo tiempo tiene impactos ambientales y sociales que deben ser considerados si no se quiere poner en peligro este bienestar a medio y largo plazo (Budeanu, 2007; Buckley, 2012). La visión de la sostenibilidad es una visión dinámica de la evolución del turismo, en la que se persigue el objetivo de conseguir que el destino turístico sea capaz de proporcionar bienestar para las generaciones futuras sin menoscabar su capacidad de generación de riqueza. Para ello, es necesario actuar en el presente de modo que los comportamientos de los turistas sean compatibles con la preservación de los recursos naturales.

El objetivo del cambio comportamental de los turistas requiere algo más que la formulación de criterios de sostenibilidad ambiental en todos los niveles de la producción y servicios (Hedlund, 2011; Tuan, 2017), pues es necesario un cambio de mayor calado en el sistema del comportamiento humano, empezando a partir de los valores, la cultura y los incentivos para tomar las decisiones. Mihalic (2016) argumenta que se necesita, además, trabajar por una triple A del turismo sostenible, es decir, la concienciación (awareness), el establecimiento de una agenda de proyectos, y la acción final o ejecución de estos proyectos, en lo que ha venido a denominarse el turismo sostenible responsable (Hall, 2013; Chong, 2013). Por tanto, en el centro de esta responsabilidad por la sostenibilidad está el consumidor turístico tomando sus decisiones habituales, tanto en origen como en destino, las cuales deben ser concienciadas ambientalmente para que actúen de forma responsable y sostenible.

En otro orden, resulta claro que el cuidado del medio ambiente y el objetivo de la sostenibilidad son principios que van en interés del bienestar humano en todos los contextos -local, regional y global-, y que solo pueden lograrse a partir de la modificación adecuada de los procesos de producción y consumo, incluidos los realizados en el contexto turístico (Waligo et al., 2013; Aall et al., 2015; Vu et al., 2016). Si todos los efectos externos entre consumidores y productores fuesen adecuadamente contemplados en la toma de decisiones individuales, es evidente que la solución serían unas pautas de consumo y producción integradas con el objetivo de la sostenibilidad. Sin embargo, el devenir turístico dista mucho de la meta de la sostenibilidad, pues aún persisten impactos ambientales poco sostenibles y comportamientos ambientales poco deseables desde el punto de vista social. 
El comportamiento del consumidor turístico no se ajusta normalmente a las predicciones trazadas por la teoría económica del consumidor racional, que formula sus decisiones basándose en la optimización de una función objetivo de satisfacción o utilidad dadas unas restricciones de renta y de tiempo (Sirakaya y Woodside, 2005; Cohen et al., 2014). La elección de productos y servicios turísticos, así como las decisiones tomadas tanto en origen como en destino, se presentan de acuerdo a la formulación tradicional, guiadas por la búsqueda racional de la máxima satisfacción individual, a partir de un cálculo de costes y beneficios que conduce a la elección o decisión óptima (Mair y Bergin-Seers, 2010). En este modelo, las elecciones del comportamiento de los turistas se pueden mejorar produciendo más satisfacción a partir del suministro de más información, mejores productos y servicios, y más opciones de elección. Sin embargo, es notorio que los turistas se desvían frecuentemente de este modelo de comportamiento racional (Dann, 1981; MacCannell, 2013), que se basa en la evaluación de los costos y beneficios de todas las alternativas posibles.

Una de las manifestaciones más recurrente de la desviación del comportamiento racional de los turistas como consumidores se observa en la disonancia entre las actitudes con respecto al medio ambiente y los comportamientos reales realizados una vez llegan al destino turístico (Cohen et al., 2013; Imran et al., 2014; Lee et al., 2014; Hall, 2016). Así, pues, los llamados valores «verdes» como el reconocer la necesidad del reciclaje, el uso de energías renovables o el consumo de productos sostenibles no se traduce en elecciones de compra proambientales de las opciones que se presentan en el mercado turístico, consistentes en la utilización de medios de transporte eficientes, el ahorro en el uso de la energía y el agua, y la elección de opciones de consumo sostenibles. Esto representa un reto desde el punto de vista de la consecución del objetivo de la sostenibilidad turística, pues sin el cambio de los comportamientos de los turistas, no es posible avanzar hacia una sociedad y una economía más consistente con las restricciones impuestas por la sostenibilidad.

Por otra parte, se han observado importantes desfases entre la acción que realizan los consumidores y turistas por un lado, y los conocimientos, los valores, las actitudes y las intenciones ambientales por otro (Juvan y Dolnicar, 2014, Karlsson y Dolnicar, 2016; Juvan et al., 2017). Así, los turistas pueden tener conocimiento, valorar, y presentar actitudes e intenciones favorables hacia comportamientos ambientales, como la minimización del consumo de agua durante su estancia en un complejo alojativo, pero comportarse de forma totalmente contraria a este objetivo. Se han aducido diversas explicaciones para esta divergencia, como son la teoría del comportamiento planeado, la teoría de la atribución, la teoría del valor-creeencia-norma, y la teoría de la disonancia cognitiva.

La teoría del comportamiento planeado es una extensión de la teoría de la acción razonada, y postula que las actitudes, las normas sociales y las percepción de controles sociales del comportamiento individual afectan las intenciones el comportamiento de los individuos en sus decisiones de consumo (Ajzen, 1985; Chen y Tung, 2014). La teoría de la atribución sostiene que las personas perciben dos causas principales de su comportamiento, las cuales interactúan entre ellas para explicar las 
decisiones tomadas: una interna centrada en la persona, y otra externa, centrada en los condicionantes sociales. Cuando las causas de las decisiones se atribuyen a los demás, o al contexto social, se limitan las decisiones de tipo individual. Por tanto, los consumidores terminan por no percibirse como causantes del daño ambiental, por lo que estiman que sus decisiones no son parte de la solución (Teng et al.2015). Este es el caso cuando se argumenta que no se pueden realizar comportamientos ambientalmente sostenibles debido a que no hay opciones para ello, o debido a que el contexto turístico invita un hedonismo de grupo que limita el efecto de la acción individual sobre el resultado ambiental.

Desde la teoría del valor-creencia-norma (Stern, 2000; Han, 2015), el comportamiento ambiental se explica por la interacción de los valores de los individuos en cuanto a los aspectos ambientales, las creencias acerca de la responsabilidad personal sobre medio ambiente y las normas personales relacionadas con el medio ambiente. Según esta teoría, las acciones reductoras de los impactos en el medio ambiente están decididas por la toma de conciencia sobre las consecuencias de las acciones, así como por creencia de una responsabilidad de la protección sobre los activos ambientales que se consideran que tienen valor (Gifford y Nilsson, 2014). Por tanto, sin un valor sobre el medio ambiente, no se puede generar una concienciación, y sin ésta resulta difícil incitar la responsabilidad que conduce a la toma de decisiones medioambientalmente responsables desde el punto de vista del consumidor individual.

La teoría de la disonancia cognitiva explica el comportamiento de los individuos con base en una contradicción entre lo que éstos consideran que tiene valor y es importante, y lo que realmente hacen basándose en los condicionantes sociales (O’Neill y Palmer, 2004; Del Bosque y San Martín, 2008). Se trata de una inconsistencia de los aspectos cognitivos (actitudes, creencias, valores, opiniones y conocimiento) percibidos para sí mismos, para el comportamiento que deben realizar y para el contexto social en que se desenvuelve este comportamiento. Esta triple divergencia genera un estado de ansiedad, incertidumbre y duda, que termina por producir insatisfacción en la elección realizada. Algunos autores sostiene que la disonancia cognitiva es medible y que existen diversos grados dependiendo del grado de divergencia entre las percepciones y las actuaciones (Tanford y Montgomery, 2015). Sin embargo, desde el punto de vista dinámico, también resulta posible que los individuos intenten reducir la disonancia cognitiva mediante el ajuste de los aspectos cognitivos (valores, creencias) o bien, corrigiendo las decisiones de comportamiento. Un ejemplo de disonancia cognitiva encontrado en las decisiones turísticas (Hares, Dickinson y Wilkes, 2010) se observa en el ingente aumento de los viajes de desplazamientos largos a pesar de la contribución demostrada de los mismos al cambio climático.

Por todo ello, debido a la dificultad de persuadir a los turistas para comportarse de una forma ambientalmente sostenible, es probable que las acciones encaminadas a concienciar a los turistas en el destino acerca de la necesidad de realizar comportamientos ambientalmente sostenibles, como las campañas de información, no produzcan los resultados esperados (Steg y Vlek, 2009; Dolnicar et al., 2017). 
Por contra, la utilización de incentivos financieros o económicos, como las tasas o exacciones por los comportamientos ambientalmente indeseables, puede conducir a la legitimización de los mismos, e incitar a un mayor impacto ambiental en contra de la sostenibilidad de los destinos, reduciendo los incentivos intrínsecos hacia un comportamiento puramente altruista.

En cierto modo, la presencia o ausencia de comportamientos proambientales de los turistas podría ser predecible en un contexto irracional (Ariely, 2008; Tufan et al., 2016), si acudimos a los resultados de las investigaciones realizadas en el ámbito de la economía del comportamiento, que sostiene que aunque los comportamientos no son racionales desde el punto de vista del interés personal y/o social, existen algunos condicionantes del proceso de elección que influyen determinantemente en estos comportamientos, por lo que son predictivamente irracionales, si observamos cómo estos condicionantes actúan en el momento de tomar las decisiones turísticas (Hall, 2013; Cohen et al., 2014). Por tanto, desde este planteamiento, el comportamiento del turista para conseguir el objetivo de la sostenibilidad está influenciado por sesgos cognitivos y tendencias de carácter irracional, que aunque producen resultados diferentes a los esperados por una elección basada en cálculos racionales, puede anticiparse y construirse con base en los resultados teóricos y empíricos de las ciencias del comportamiento humano.

Algunos de estos sesgos en el comportamiento de los turistas, y que producen desviaciones de comportamiento con respecto al modelo de comportamiento racional, emergen de la utilización de sencillas reglas de decisión, heurísticas, y atajos mentales que reducen el esfuerzo requerido para resolver el complejo problema de optimización de la satisfacción individual (Oechssler et al., 2009; Hoppe y Kusterer, 2011; Hsu y Huang, 2012). Se trata de artimañas que los consumidores turistas utilizan para sortear la necesidad de organizar cantidades ingentes de información, acelerando la toma de decisiones y la solución del problema de elección, particularmente en situaciones caracterizadas por una alta complejidad, muchas posibilidades de elección, riesgo e incertidumbre. Algunos de los sesgos que más influyen en la elección de los turistas en sus decisiones ambientales son: 1) el efecto del status quo, 2) aversión al riesgo y a las pérdidas, 3) los costes hundidos, 4) el descuento espacial y temporal y 5) el sesgo de la disponibilidad. Otros condicionantes psicológicos y sociales que también influyen determinantemente en la elección de opciones proambientales son: 6) las normas sociales, 7) la recompensa intrínseca y extrínseca y 8) la confianza.

\section{Diseño experimental}

\subsection{Emisiones de $\mathrm{CO}_{2}$}

El experimento de campo para las elecciones de emisiones de $\mathrm{CO}_{2}$ se implementó con clientes de una agencia de viajes, dispuestos a comprar paquetes turísticos. El 
número total de encuestados válidos para el estudio fue de 269. Se contactó a los sujetos en la entrada de las oficinas de la agencia de viajes y se les solicitó participar en el estudio a cambio de algunos regalos útiles para su viaje. La tasa de participación fue del 66 por 100 del total de personas contactadas.

Este experimento presenta la ventaja que se trata de un experimento de campo, $\mathrm{y}$ por tanto los participantes toman decisiones en su vida diaria, con lo que se trata de decisiones plenamente consecuentes, evitando el sesgo proporcionado por los estudios de actitudes, cuyas estimaciones pueden diferir significativamente de las decisiones reales -brecha actitud-comportamiento- (Juvan y Dolnicar, 2014; Antimova et al., 2012; Font et al., 2012).

En este experimento, el equipo de encuestadores seleccionaba a los participantes aleatoriamente a su llegada a la agencia de viajes, y los distribuía en cinco grupos de tratamiento dependiendo de la información suministrada. Una vez se le daba la bienvenida a los participantes, se les pedía firmar una hoja de consentimiento para el experimento. Seguidamente se les pedía responder a un cuestionario general sobre calidad de vida, para después ser asignados aleatoriamente a los cinco tratamientos alternativos, que son los siguientes:

\section{Grupo de control}

Este grupo recibió una intervención neutral en la que se les mostró un videoclip de National Geographic sobre la Gran Barrera de Coral, intencionalmente seleccionado con el fin de despertar emociones neutrales. Además, este grupo no recibió ninguna propuesta de política ambiental.

\section{Impuestos ambientales}

El grupo 2 recibió un tratamiento de impuestos ambientales ${ }^{2}$ sobre el precio final del paquete turístico elegido de acuerdo a las emisiones de $\mathrm{CO}_{2}$. Los individuos en este grupo también observaron el mismo video que el grupo 1, pero una vez finalizada la emisión, elegían los paquetes turísticos sujeto a un incremento del precio de acuerdo a una tasa impositiva que variaba aleatoriamente entre los valores de 5 por 100 y 20 por 100. El impacto de estas tasas de impuestos alternativas sobre las opciones de los turistas y los niveles de emisiones de $\mathrm{CO}_{2}$ asociados, permite probar la eficacia de dicha política de sostenibilidad para reducir los niveles promedio de emisiones de $\mathrm{CO}_{2}$ a través de la modificación del comportamiento mediante la elección.

\footnotetext{
${ }^{2}$ Algunos trabajos que estudian el impacto de impuestos ambientales sobre el comportamiento del turista son BIRD (1992), CLARK y NG (1993), DIMANCHE (2003), GOSSLING et al. (2007) y PALMER-TOUS et al. (2007).
} 


\section{Etiquetas de impactos de $\mathrm{CO}_{2}$}

El grupo 3 seguía el mismo tratamiento que los grupos 1 y 2, pero iba acompañado de un etiquetado ${ }^{3}$ con la cantidad de emisiones involucradas en cada opción a elegir (Gössling y Buckley, 2016). El fundamento de esta política es que la información puede conducir a aumentar la conciencia de los turistas, induciendo un cambio activo en el comportamiento hacia opciones de paquetes turísticos más sostenibles.

\section{Presión de tiempo}

Este grupo recibió el mismo tratamiento que los grupos 1 y 2 , pero se les conminaba a elegir entre las alternativas en un periodo de tiempo restringido o limitado, de modo que la decisión se tomaba bajo una presión temporal. En el momento de tomar la decisión de elección, a los sujetos se les recordaba que los destinos y precios estaban disponibles para ellos solo en ese momento, y si no compraban el paquete inmediatamente ya no había garantía de tener la posibilidad de viajar esas alternativas a esos precios ${ }^{4}$. Cabe esperar que lo sujetos sometidos a este tratamiento muestren una mayor probabilidad de viajar (aumentar el número de viajes), conduciendo a un aumento del nivel de emisiones de $\mathrm{CO}_{2}$.

\section{Manipulaciones emocionales}

El grupo 5 siguió el mismo tratamiento que los grupos 1 y 2 , y se dividió a su vez en dos subgrupos aleatoriamente, en función del videoclip proporcionado antes de enfrentarse a las opciones de elección de viajes alternativos. Estas dos alternativas de tratamiento pretendían suscitar cuatro grupos de emociones diferentes en los participantes: tristeza, empatía, enfado y felicidad. Para la invocación de tristeza se escogió el videoclip oficial del Panel Internacional para el Cambio Climático (IPCC). Para la suscitación de empatía se escogió el videoclip de la reunión de las Naciones Unidas en Río, donde una niña (Severin Suzuki) explicó por qué se necesitaban políticas internacionales para el cambio climático. Para la generación de una emoción de enfado, se utilizó un fragmento de la película de Leonardo DiCaprio titulada Antes de la inundación, que intenta despertar en el espectador la inminente necesidad de actuar para solucionar el problema del cambio climático. Para la generación de un estado de felicidad en relación a los viajes, se escogió la película «Come, Ora y Ama» protagonizada por Julia Roberts en 2010, en la cual la protagonista descubre que los viajes

\footnotetext{
${ }^{3}$ La provisión de información ambiental (etiquetado de emisiones de $\mathrm{CO}_{2}$ ) es una de las políticas de sostenibilidad más extendidas (LUCAS et al., 2008).

${ }^{4}$ Existe evidencia que las prácticas de presión temporal aumenta la percepción de valor del consumidor, conllevando mayores intenciones de compra (VERMEIR y VAN KENHOVE, 2005) y aumentando la probabilidad de elección (INMAN et al., 1997).
} 
por varios continentes proporcionan el sentido que buscaba en su vida. Todos los videos tuvieron una duración similar, y fueron elegidos después de un extenso trabajo cualitativo previo a la encuesta, en el que se demostró su capacidad para suscitar las emociones pretendidas por la investigación en los participantes.

\subsection{Reutilización de enseres}

El experimento 2 se centra en los mensajes para incitar a los turistas a reutilizar las toallas y la ropa de cama en los establecimientos alojativos. Los mensajes informan a los turistas alojados acerca de la opción de la reutilización, invocando objetivos ambientales. Se trata de una medida normativa y de persuasión dirigida a modificar el comportamiento ambiental del turista.

Se seleccionaron 4 establecimientos extrahoteleros, con un número medio de 41 habitaciones, los cuales no habían emprendido anteriormente ninguna medida de información persuasiva en las habitaciones. El número total de habitaciones fue de 164. Los establecimientos fueron escogidos por su homogeneidad en cuanto a la política de cambio de las toallas y sábanas. Para el experimento se acordó que las toallas se cambiasen diariamente y las sábanas cada tres días, si el cliente no solicitaba expresamente su reutilización a través de los canales determinados en el experimento. Para cada establecimiento se distribuyeron aleatoriamente los mensajes persuasivos en las habitaciones y se observaron los comportamientos de los turistas alojados en cuanto a la reutilización o no de las toallas y ropa de cama.

Se distribuyeron dos mensajes alternativos de acuerdo a la cantidad de información proporcionada sobre las consecuencias ambientales de la reutilización de las toallas y sábanas. Para el grupo de menor información, el mensaje fue el siguiente:

Estimado cliente,

De acuerdo con nuestro compromiso de cuidar el medio ambiente y reducir el uso del preciado recurso del agua, le ofrecemos las siguientes opciones durante su estancia:

1) Coloque su toalla en la bañera/ducha para el lavado diario si quiere que se la cambien (servicio normal).

2) Cuelgue sus toallas en el toallero si decide dejarlas secar para volverlas a usar.

Las sábanas en este establecimiento se cambian cada 3 días. Si desea cambiar su ropa de cama, coloque este letrero en su cama. En [establecimiento alojativo] apoyamos la interacción sostenible con la naturaleza y el uso responsable del agua, que es un recurso de alto valor, cada vez más escaso en nuestro planeta. 
La mayoría de los huéspedes alojados en este establecimiento han decidido volver a utilizar las toallas, al menos durante unos días, durante su estancia. Lo mismo ha ocurrido con la ropa de cama. El ahorro de costes que estas medidas supondrá para [establecimiento alojativo] se destinará a una organización ambiental no gubernamental (ONG) dedicada a la implementación de medidas para el ahorro de agua en el mundo, así como para la mejora de su suministro en zonas de escasez aguda.

Para el grupo de más información, se añadía el siguiente párrafo informativo:

El recambio de las toallas y la ropa de cama tiene impactos importantes en el medio ambiente y en el consumo de agua. Por una parte, el uso de detergentes mediante el lavado industrial genera vertidos de contaminantes químicos al medio, que pueden terminar por dañar los acuíferos de aguas subterráneas, y afectar a la salud humana a través de la contaminación del agua y la ingesta de productos agrícolas en suelos contaminados. Por otra parte, el lavado industrial también supone un consumo de energía que contribuye a las emisiones de $\mathrm{CO}_{2}$ que provocan el cambio climático. Además, el uso del agua contribuye a reducir la disponibilidad de este valioso recurso. Por ejemplo, por cada día que se reutilizan sus toallas y ropa de cama se ahorran un total de 30 litros de agua. Muchas gracias por APOYAR EL CUIDADO DEL MEDIO AMBIENTE.

Por otra parte, debido que algunos clientes pueden no prestar demasiada atención a las etiquetas con información incorporadas en las habitaciones, las dos submuestras, tanto con mayor o menor información, se distribuyeron a su vez en otras dos submuestras en función de si se informaba a los clientes en recepción acerca de la presencia de las etiquetas o no.

Siguiendo las recomendaciones de la literatura, el mensaje iba acompañado del logotipo del hotel (Shang et al., 2010) y recordaba que la mayoría de los turistas alojados estaban de acuerdo en la reutilización de las toallas y sábanas (Goldstein et al., 2008; Van der Linden, 2014). Asimismo, se establece una regla de reciprocidad, en el sentido que el establecimiento dedica los ahorros de agua y energía obtenidos con el cambio de comportamiento a una organización benéfica, esperando, por tanto, la misma respuesta por parte de los huéspedes (Goldstein et al., 2011).

El mensaje se colocó en tres idiomas (alemán, inglés y español) en un sitio visible y distinguido en la habitación, así como en el cuarto de baño, de manera que generó la atención inmediata de los huéspedes. En el texto del mensaje se incluye información clara de qué se debe hacer para reutilizar las toallas y sábanas. Se realizaron trabajos previos con reuniones de grupo, en las que participaron dos grupos de cuatro turistas (inglés y alemán), que permitieron probar que los mensajes eran entendidos perfectamente por los clientes potenciales de las habitaciones.

A lo largo de un periodo de tres meses, se realizaron observaciones de los clientes alojados en las habitaciones en los que se habían incorporado aleatoriamente los dos 
tipos de mensaje de acuerdo a la cantidad de información. La observación consistió en los datos socioeconómicos de los turistas alojados (edad, género, nacionalidad, estadía), obtenidos de la ficha de registro a la entrada del establecimiento, así como la decisión realizada en cuanto a la reutilización de los enseres durante la estancia en la habitación, y el tiempo en que fueron reutilizados. Los clientes que pernoctaron solo una noche en el establecimiento fueron excluidos de las muestras obtenidas. En total, se obtuvieron 1.968 observaciones de turistas alojados, de los cuales 987 correspondían al grupo de menor información y 981 al grupo de mayor información.

Los datos sobre la reutilización de los enseres fueron recabados por el personal de limpieza. El personal fue informado sobre el propósito del experimento y capacitado en la recolección de datos, es decir, cuándo registrar las toallas reemplazadas. Esto implicó el cambio de toallas y ropa de cama durante el servicio de limpieza de la habitación. Los datos debían ingresarse en una matriz de datos diaria de las habitaciones desarrollada específicamente para este propósito, y distribuidos diariamente al personal.

\section{Resultados}

\subsection{Emisiones de $\mathrm{CO}_{2}$}

Las submuestras utilizadas en este experimento no presentaron características socioeconómicas diferentes entre sí, por lo que se puede esperar que las diferencias en cuanto a los comportamientos observados se deben solo al tratamiento experimental de cada grupo.

Dado que un aspecto importante de la toma de decisiones en este experimento es el estado emocional de los participantes, el Cuadro 1 presenta los resultados de las autoevaluaciones de las emociones de los viajeros en los diferentes grupos de tratamiento, una vez que les fueron presentados los vídeos respectivos a los participantes. Los grupos 1, 2, 3 y 4 fueron sometidos a un tratamiento de emociones neutrales, o indiferentes, mientras que el grupo 5 fue sometido a una manipulación para suscitar cuatro tipos de emociones diferentes: tristeza, empatía, enfado y felicidad. Como puede verse en el Cuadro 1, los tratamientos de suscitación de las emociones funcionaron adecuadamente en los encuestados, de modo que cabe esperar con un alto nivel de confianza que los individuos tomaron las decisiones de elección entre paquetes turísticos alternativos con distintos niveles de emisiones de $\mathrm{CO}_{2}$ bajo los estados emocionales pretendidos por los tratamientos experimentales.

Una vez los individuos han elegido los paquetes turísticos alternativos y mostrado, por tanto, su disposición a pagar por los mismos, se pueden evaluar los impactos de emisiones medias de $\mathrm{CO}_{2}$ por persona que resultan de estas elecciones. El Gráfico 1 presenta los niveles promedio de emisión de $\mathrm{CO}_{2}$ (toneladas métricas por persona) para cada tratamiento experimental, tanto si se trata de medidas de política impositiva (incentivos económicos), como de provisión de información de emisiones (etiquetas) o la manipulación del estado emocional. El grupo 1 fue el 
grupo de control en el que se empleó el proceso de reserva convencional, en el que no se aplica ninguna medida disuasoria de las elecciones que involucren elevados niveles de $\mathrm{CO}_{2}$, ni se despiertan estados emocionales que pueden alterar la elección. Los niveles de emisión observados para este grupo pueden utilizarse como referencia para comparar el impacto de diferentes tratamientos experimentales. Así, para el grupo neutral, los resultados muestran que el nivel promedio de emisiones de $\mathrm{CO}_{2}$ fue de 0,55 toneladas métricas por persona.

\section{CUADRO 1}

EMOCIONES SUSCITADAS POR CADA TRATAMIENTO EXPERIMENTAL

\begin{tabular}{|c|c|c|c|c|}
\hline & \multicolumn{4}{|c|}{ Emoción suscitada (escala 1-5) } \\
\hline Tratamiento & Tristeza & Empatía & Enfado & Felicidad \\
\hline Neutral & 1,1 & 1,3 & 1,4 & 1,3 \\
\hline Tristeza & $\mathbf{4 , 3}$ & 1,4 & 1,3 & 1,2 \\
\hline Empatía & 1,2 & $\mathbf{4 , 8}$ & 1,2 & 1,1 \\
\hline Enfado & 1,2 & 1,4 & $\mathbf{4 , 7}$ & 1,2 \\
\hline Felicidad & 1,3 & 1,5 & 1,1 & $\mathbf{4 , 6}$ \\
\hline
\end{tabular}

FUENTE: Elaboración propia.

\section{GRÁFICO 1}

EMISIONES POR PERSONA (TM/PERSONA) SEGÚN TRATAMIENTO EXPERIMENTAL

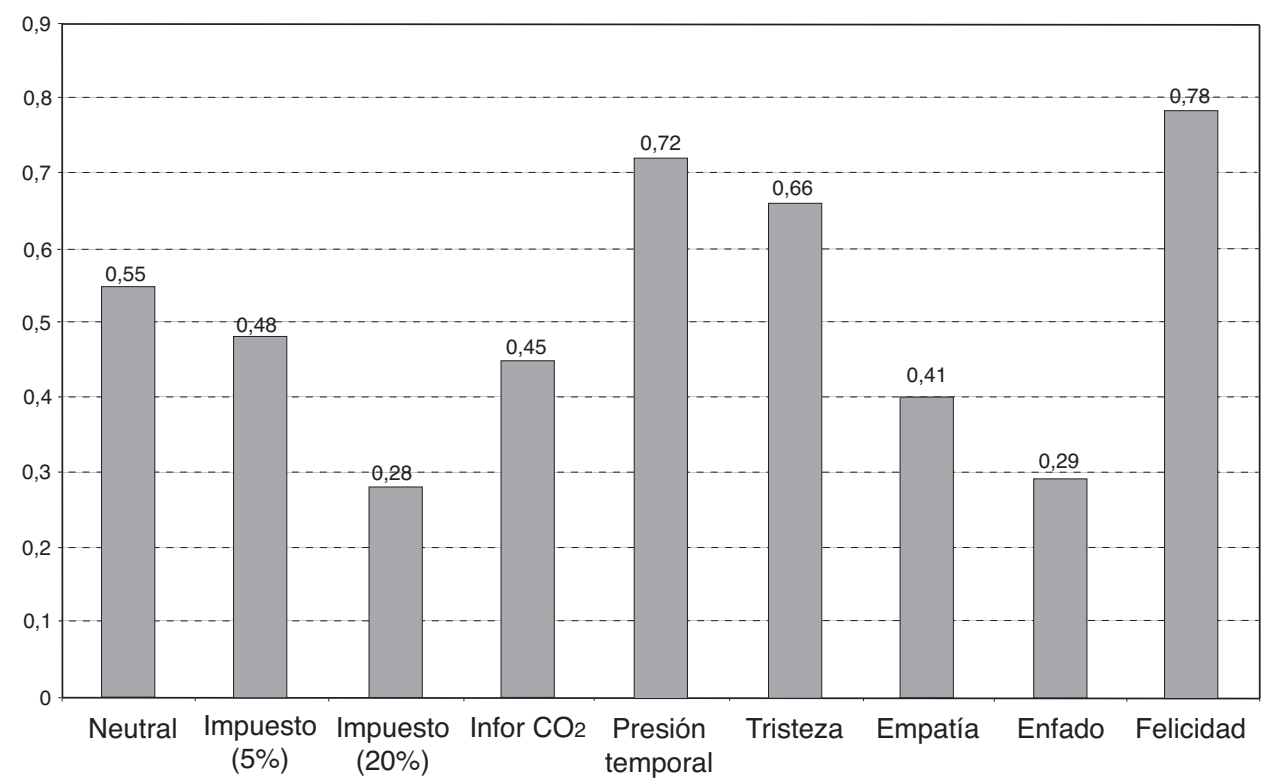

FUENTE: Elaboración propia. 
Las medidas de intervención introducidas en los grupos alternativos dan lugar a distintos niveles de emisiones de $\mathrm{CO}_{2}$ con respecto al grupo de control que recibió el tratamiento neutral. El Cuadro 2 muestra el cálculo de los porcentajes de variación de las emisiones para cada tratamiento alternativo con respecto al grupo de control. Para la política de aplicar una tasa impositiva al impacto de emisiones de $\mathrm{CO}_{2}$, se observa una reducción importante de las emisiones, hasta un nivel de 0,48 con una tasa del 5 por 100, y un nivel de 0,28 con una tasa del 20 por 100. Por tanto, este resultado indica que la aplicación de una tasa impositiva a las emisiones de $\mathrm{CO}_{2}$ sobre los paquetes turísticos elegidos tiene un efecto disuasorio en la elección de aquellos paquetes que conllevan mayor cantidad de emisiones, y que cuanto mayor es la tasa más importante es la reducción de emisiones. Para una tasa del 20 por 100 la reducción de emisiones es del 49 por 100, mientras que para una tasa del 5 por 100 la reducción es tan solo del 12,7 por 100.

\section{CUADRO 2}

VARIACIÓN DE EMISIONES DE $\mathrm{CO}_{2}$ (En \%)

\begin{tabular}{|l|c|}
\hline \multicolumn{1}{|c|}{ Tratamiento } & Variación \\
\hline Impuesto $(5 \%)$ & $-12,7$ \\
\hline Impuesto $(20 \%)$ & -49 \\
\hline Infor $\mathrm{CO}_{2}$ & $-18,18$ \\
\hline Presión temporal & 30,9 \\
\hline Tristeza & 20 \\
\hline Empatía & $-25,45$ \\
\hline Enfado & $-47,27$ \\
\hline Felicidad & 41,81 \\
\hline
\end{tabular}

FUENTE: Elaboración propia.

Por otra parte, la política de incorporar información sobre las emisiones de $\mathrm{CO}_{2}$ de cada alternativa de elección también tiene un efecto significativo en la reducción de las emisiones de los paquetes elegidos, hasta un nivel de 0,45 por turista, es decir, de un 18,2 por 100. Por tanto, esta medida tiene un efecto similar que la aplicación de impuestos moderados en la reducción de las elecciones que conducen a mayores emisiones per cápita. En esta línea, algunos autores (Becken, 2007; Gössling et al., 2008) han sugerido que la falta de información concreta sobre las emisiones de $\mathrm{CO}_{2}$ generadas por cada decisión de viaje específica es una causa fundamental de algunos comportamientos de movilidad insostenibles observados en las decisiones turísticas. Por ello, la provisión de información clara y precisa para cada decisión de viaje puede reducir significativamente los niveles de emisión de $\mathrm{CO}_{2}$. 
Una de las técnicas de venta más extendidas es la oferta de productos y servicios bajo un tiempo limitado, de modo que se acelere la toma de decisión por parte del consumidor. En este caso, el individuo puede no tener el tiempo suficiente para evaluar con reflexión todas las alternativas, y por tanto, puede tender a utilizar reglas de decisión más simples, que dan una mayor ponderación a los atributos más sobresalientes, como el precio y la marca del destino, y una menor ponderación a los atributos que tienen una mayor dificultad para ser entendidos, como el impacto ambiental de las decisiones de viaje. Como se puede apreciar en el Gráfico 1 y en el Cuadro 2, el tratamiento bajo presión temporal condujo a un aumento significativo de las emisiones medias hasta un 0,72 en promedio, esto es, un aumento del 31 por 100. Este resultado sugiere que cuanto más tiempo tengan los sujetos para tomar las decisiones de elección de viajes con distintos niveles de emisiones de $\mathrm{CO}_{2}$, menor será el volumen de emisiones agregadas.

En cuanto al impacto de las manipulaciones emocionales en los niveles de emisión de $\mathrm{CO}_{2}$, se observa en el Gráfico 1 y en el Cuadro 2 que tanto la tristeza como la felicidad conducen a aumentos significativos de las emisiones, con un aumento del 20 por 100 para la tristeza ${ }^{5}$-hasta $0,66-$ y del 41,8 por 100 para la felicidad. Sin embargo, la empatía y el enfado dan lugar a la elección de alternativas de viaje con menores niveles de emisiones de $\mathrm{CO}_{2}$ por persona. En el caso de la empatía, la reducción es del 25,4 por 100 y para el enfado la reducción es del 47,2 por 100. Por tanto, los mensajes conducentes a incentivar emocionalmente las variaciones de emisiones de $\mathrm{CO}_{2}$ en los procesos de compra de viajes turísticos deberían tener en cuenta que los estados emocionales asociados a estas elecciones son relevantes, con una mayor incidencia en el aumento de las emisiones si se trata de mensajes de felicidad, y una reducción mayor en el caso de mensajes de enfado.

\subsection{Reutilización de enseres}

Las cuatro submuestras utilizadas en este experimento no presentaron diferencias estadísticamente significativas en cuanto a las variables socioeconómicas de edad, género, repetición de las visitas y nacionalidades, que se definen en el Cuadro 3, y que se utilizan para modelizar la decisión de reutilizar o no las toallas y la ropa de cama, cuyos resultados se presentan en el Cuadro 4.

La variable dependiente para la modelización indica el grado o índice de reutilización escogido por el cliente. Esto es, se trata de una variable construida como el cociente entre el número de días (o veces) que el cliente ha decidido optar por la reutilización y el número de días totales de su estancia en los que se le ha podido realizar el recambio. La variable toma un valor entre 0 y 1 , siendo 0 cuando el cliente no opta en ningún caso por la reutilización, y 1 cuando durante todos los días de su

\footnotetext{
${ }^{5}$ Algunas campañas prosostenibles (por ejemplo, el vídeo del IPCC) han demostrado que inducen a la tristeza y pueden desincentivar involuntariamente el comportamiento sostenible (MOSER, 2014).
} 
estancia en los que se realiza cambio de toallas y/o ropa de cama, decide optar por la reutilización. Por tanto, para el valor igual a 1 de la variable dependiente, el cliente no ha cambiado estos enseres durante toda su estancia, con lo que las medidas de persuasión para el ahorro de agua han tenido su mayor impacto.

\section{CUADRO 3}

\section{DEFINICIÓN DE LAS VARIABLES EXPLICATIVAS DE LA REUTILIZACIÓN DE TOALLAS Y ROPA DE CAMA}

\begin{tabular}{|l|l|}
\hline \multicolumn{1}{|c|}{ Variables } & \multicolumn{1}{c|}{ Definición } \\
\hline Información & $\begin{array}{l}\text { Toma el valor } 1 \text { si el sujeto es ha recibido las etiquetas con más información } \\
\text { y } 0 \text { en caso contrario. }\end{array}$ \\
\hline Recepción & $\begin{array}{l}\text { Toma el valor 1 si el sujeto fue informado del contenido de las etiquetas en } \\
\text { recepción y 0 en caso contrario. }\end{array}$ \\
\hline Edad & Años de edad del individuo más adulto en la habitación. \\
\hline Estancia & Número de días de la estancia en el establecimiento alojativo. \\
\hline Repetición & Número de veces que el cliente ha estado anteriormente alojado. \\
\hline Escandinavo & Toma el valor 1 si el sujeto es de un país escandinavo y 0 en caso contrario. \\
\hline Alemán & Toma el valor 1 si el sujeto es de Alemania y 0 en caso contrario. \\
\hline Reino Unido & Toma el valor 1 si el sujeto es del Reino Unido y 0 en caso contrario. \\
\hline Español & Toma el valor 1 si el sujeto es de España y 0 en caso contrario. \\
\hline
\end{tabular}

FUENTE: Elaboración propia.

CUADRO 4

MODELO DE REGRESION LINEAL DEL ÍNDICE DE REUTILIZACIÓN

\begin{tabular}{|l|c|c|c|c|}
\hline \multirow{2}{*}{ Variables } & \multicolumn{2}{c|}{ Toallas } & \multicolumn{2}{c|}{ Ropa de cama } \\
\cline { 2 - 5 } & Parámetro & Error estándar & Parámetro & Error estándar \\
\hline Constante & $1,672^{*}$ & 0,291 & $1,031^{*}$ & 0,198 \\
\hline Información & 0,172 & 0,141 & $0,381^{*}$ & 0,058 \\
\hline Recepción & $0,305^{*}$ & 0,132 & $0,611^{*}$ & 0,135 \\
\hline Edad & $-0,074^{*}$ & 0,027 & $-0,054$ & 0,047 \\
\hline Estancia & $0,065^{*}$ & 0,021 & $0,048^{*}$ & 0,022 \\
\hline Estancia ${ }^{2}$ & $-0,003^{*}$ & 0,001 & $-0,069$ & 0,052 \\
\hline Repetición & $0,138^{*}$ & 0,055 & 0,265 & 0,139 \\
\hline Escandinavo & $0,472^{*}$ & 0,128 & $0,361^{*}$ & 0,075 \\
\hline Alemán & $-0,390^{*}$ & 0,153 & 0,252 & 0,180 \\
\hline Inglés & $-0,141$ & 0,133 & 0,121 & 0,160 \\
\hline Español & $-0,442^{*}$ & 0,172 & $0,283^{*}$ & 0,020 \\
\hline $\mathrm{R}^{2}$ & \multicolumn{2}{|l}{0,58} \\
\hline
\end{tabular}

NOTA: * Significativo al 95 por 100.

FUENTE: Elaboración propia. 
Como se puede observar en el Cuadro 4, el índice de reutilización es significativamente mayor para los individuos que han recibido información en la recepción, tanto en la decisión de reutilizar las toallas como la ropa de cama, dado que la variable recepción toma valor positivo. Sin embargo, la variable información es solo significativa para la reutilización de ropa de cama. Por tanto, la incorporación de mayor información en las etiquetas de las habitaciones solo surte un efecto positivo en la reutilización para el caso de la ropa de cama, y no para las toallas. Este resultado se compensa con la provisión de información en la recepción, que tiene un mayor impacto en la reutilización que la proporcionada en las etiquetas de las habitaciones.

La tasa de reutilización es menor para las habitaciones que alojan individuos de mayor edad en el caso de la reutilización de las toallas, dado que la variable edad es negativa y significativa al 95 por 100 para este modelo. Para el modelo de la reutilización de la ropa de cama, la edad del individuo más adulto, aunque tiene signo negativo no resulta significativa. Por tanto, es de esperar que las habitaciones con individuos de mayor edad tengan una menor tendencia a la reutilización de las toallas, aunque en la ropa de cama la edad de los individuos en la habitación no es una variable condicionante en la decisión de reutilización.

Por otra parte, los individuos que realizan una mayor estancia en el complejo alojativo presentan también una mayor tasa de reutilización de las toallas y de la ropa de cama, puesto que la variable estancia es significativa al 95 por 100. Sin embargo, la forma cuadrática de esta variable solo es significativa para la tasa de reutilización de las toallas. Esto es, la reutilización de toallas aumenta con la estancia, pero tiende a decrecer para estancias más largas. Esta eventual reducción de la reutilización con la duración de la estancia no ocurre con la ropa de cama, ya que la variable cuadrática no es significativa para este modelo.

En cuanto a los clientes que ya han estado anteriormente en el complejo alojativo, se observa que tienden a una mayor reutilización de las toallas, pero no de la ropa de cama. Así, la variable repetición es positiva y significativa al 95 por 100 solo para el modelo de reutilización de las toallas, por lo que la mayor reutilización para clientes fidelizados no se observa para las decisiones sobre la ropa de cama.

La tasa de utilización también varía significativamente entre los clientes de distintas nacionalidades, lo cual puede estar motivado por razones culturales, de hábitos turísticos o de sensibilidad ambiental. Así, se observa que la tasa de reutilización es mayor para los turistas escandinavos, tanto en el caso de las toallas como en la ropa de cama, y menor para los turistas alemanes y españoles, pero solo para las toallas. Los turistas españoles tienden, sin embargo, a presentar una mayor tasa de utilización de la ropa de cama. En el caso de los turistas ingleses, no se observa ninguna diferencia significativa con respecto a la media de los turistas en la reutilización ni de las toallas ni de la ropa de cama.

El Cuadro 5 presenta la proporción de reutilización de toallas y ropa de cama para los cuatro tratamientos muestrales considerados en este experimento. Como puede apreciarse, la tasa o índice de reutilización es en general más alta para el caso de las toallas que para la ropa de cama. Para el escenario de baja información en la habita- 


\section{CUADRO 5 \\ ÍNDICE DE REUTILIZACIÓN PARA CADA TRATAMIENTO MUESTRAL \\ (En \%)}

\begin{tabular}{|l|c|c|}
\hline \multirow{2}{*}{} & \multicolumn{2}{|c|}{ Toallas } \\
\cline { 2 - 3 } & 33,5 & 51,2 \\
\hline Información $=0$ & 38,7 & 52,4 \\
\hline Información $=1$ & \multicolumn{2}{|c|}{ Ropa de cama } \\
\hline & 12,1 & 28,9 \\
\hline Información $=0$ & 22,8 & 35,5 \\
\hline Información $=1$ & & \\
\hline
\end{tabular}

FUENTE: Elaboración propia.

ción y de ausencia de instrucciones informativas en recepción, la tasa de utilización de las toallas es del 33,5 por 100 y la de la ropa de cama del 12,1 por 100 . Sin duda, este resultado indica que los turistas son más proclives a reutilizar las toallas que la ropa de cama, tal vez motivados por las diferencias involucradas en la reutilización en ambos tipos de enseres.

La provisión de información en la recepción presenta un alto impacto en la reutilización de las toallas y de la ropa de cama. Este resultado indica que el impacto de la medida de etiquetado en las habitaciones puede ser reforzado con una mayor persuasión de los clientes hacia comportamiento de reutilización de los enseres. El impacto de la información incorporada en las habitaciones es, sin embargo, mucho más moderado que el impacto de la información en la recepción, siendo prácticamente irrelevante para la inducción de una mayor reutilización de las toallas.

\section{Conclusiones}

La industria turística presenta en su devenir histórico el inexcusable reto de la sostenibilidad, que implica su desarrollo a partir del principio de ser capaz de generar bienestar a medio y largo plazo con los recursos humanos y naturales disponibles, tanto en el presente como en el futuro. Este objetivo solo puede lograrse si los recursos naturales son gestionados de forma que se conserven en su estado de máxima calidad ambiental, optimizando su utilización para obtener el mejor rendimiento de los mismos, evitando por tanto su degradación o su eliminación.

La evolución observada del turismo en el contexto de su contribución al cambio climático induce a dudar seriamente acerca de su sostenibilidad con el presente statu quo de usos tecnológicos en la producción y utilización de energía, tanto a nivel local como global, si no se adoptan cuanto antes medidas que favorezcan una transición a unos procesos de producción y consumo con energías limpias y renovables. 
En este contexto de una necesidad de cambio en los sistemas de producción y consumo turístico, el turista como consumidor tiene un papel esencial en la adopción de decisiones de consumo más responsables y sostenibles, que induzcan a las organizaciones turísticas a adoptar los cambios que la demanda exige desde el punto de vista de las preferencias ambientales. En este punto, resulta por tanto muy relevante conocer cómo se comporta el consumidor turístico, como elemento central del sistema turístico, en la adopción de decisiones que tienen impacto ambiental, para a partir de este conocimiento, diseñar las medidas de política adecuadas que aceleren la transición hacia una economía turística responsablemente más sostenible.

Sin embargo, dado que el turismo es una industria por definición caracterizada por la venta de sensaciones hedonistas, y donde la búsqueda del placer es el objetivo principal del consumo, está resultando una ingente tarea la de inculcar en los turistas patrones de comportamiento que impliquen restricciones en la obtención de experiencias placenteras que no conlleven consecuencias ambientalmente negativas, observándose como resultado una prevalencia generalizada de comportamientos excesivamente descuidados del ahorro de los recursos ambientales, como el agua o la energía, en los procesos turísticos.

En cierto modo, el turista parece desconectar de su modus operandi cuando se plantea y realiza el desplazamiento a un lugar distinto a su residencia habitual, y tiende a llevar a cabo en el destino comportamientos disonantes con sus actitudes o valores ambientales. La reconducción de estos comportamientos hacia decisiones ambientalmente más responsables y sostenibles es una tarea que requiere el diseño de medidas que incentiven a los turistas a tomar decisiones más coherentes con sus valores y actitudes, reduciendo por tanto la observada brecha con los comportamientos realizados.

La formulación de medidas eficaces y eficientes conducentes a modificar los comportamientos de los turistas hacia decisiones más sostenibles debe tener en cuenta todos los aspectos que influyen en la toma de decisiones. El modelo convencional basado en la consideración de los factores económicos como el precio, debe ser ampliado para la consideración de otros condicionantes como las emociones, el contexto social, la concienciación y las normas sociales.

En este trabajo se han presentado resultados de dos experimentos que proporcionan evidencia acerca de la influencia que tiene los aspectos cognitivos y emocionales en las decisiones acerca de la elección de viajes turísticos con distintos niveles de emisión de $\mathrm{CO}_{2}$, y del ahorro de agua en los establecimientos alojativos. La evidencia aportada por ambos experimentos permite concluir que las políticas de precios a través de impuestos pueden ser tan relevantes como las políticas de sensibilización social y de provisión de información, así como la posible inducción de estados emocionales proclives a la adopción de decisiones responsables con el medio ambiente y la sostenibilidad. 


\section{Referencias bibliográficas}

[1] AALL, C.; DODDS, R.; SÆLENSMINDE, I. y BRENDEHAUG, E. (2015). «Introducing the concept of environmental policy integration into the discourse on sustainable tourism: a way to improve policy-making and implementation?». Journal of Sustainable Tourism, 23 (7), 977-989.

[2] AHO, S. K. (2001). «Towards a general theory of touristic experiences: Modelling experience process in tourism». Tourism Review, 56 (3/4), 33-37.

[3] AJZEN, I. (1985). «From Intentions to Actions: A Theory of Planned Behavior». En J. Kuhl y J. Beckmann (eds.), Action Control: From Cognition to Behavior, 11-39. Berlín: Springer-Verlag.

[4] ANTIMOVA, R.; NAWIJN, J. y PEETERS, P. (2012). «The awareness/attitude-gap in sustainable tourism: a theoretical perspective». Tourism Review, 67 (3), 7-16.

[5] ARIELY, D. (2008). Predictably Irrational (p. 20). Nueva York: HarperCollins.

[6] BACA-MOTES, K.; BROWN, A.; GNEEZY, A.; KEENAN, E. A.y NELSON, L. D. (2013). «Commitment and Behavior Change: Evidence from the Field». Journal of Consumer Research, 39 (5), 1070-84.

[7] BARSKY, J. y NASH, L. (2002). «Evoking Emotion: Affective Keys to Hotel Loyalty». Cornell Hotel and Restaurant Administration Quarterly, 43 (1), 39-46.

[8] BECKEN, S. (2007). «Tourists' perception of international air travel impact on the global climate and potential climate change policies». Journal of Sustainable Tourism, $15,351-368$.

[9] BIRD, R. (1992). «Taxing tourism in developing countries». World Development, 20 (8), 1145-1158.

[10] BUCKLEY, R. (2012). «Sustainable tourism: Research and reality». Annals of Tourism Research, 39 (2), 528-546.

[11] BUTLER, R. W. (1999). «Sustainable tourism: A state-of-the-art review». Tourism Geographies, 1 (1), 7-25.

[12] CHEN, M.F. y TUNG, P. J. (2014). «Developing an extended theory of planned behavior model to predict consumers' intention to visit green hotels». International Journal of Hospitality Management, 36, 221-230.

[13] CHONG, K. S. (2013). Cases in Sustainable Tourism: An Experiential Approach to Making Decisions. Routledge.

[14] CLARK, H. y NG, Y.(1993). «Tourism, economic welfare and efficient pricing». Annals of Tourism Research, 20 (4), 613-632.

[15] COHEN, S. A. y HIGHAM, J. E. (2011). «Eyes wide shut? UK consumer perceptions on aviation climate impacts and travel decisions to New Zealand». Current Issues in Tourism, 14 (4), 323-335.

[16] COHEN, S. A.; PRAYAG, G. y MOITAL, M. (2014). «Consumer behaviour in tourism: Concepts, influences and opportunities». Current Issues in Tourism, 17 (10), 872-909.

[17] COHEN, S. A.; HIGHAM, J. E.; STEFAN, G. y PEETERS, P. (eds.). (2014). Understanding and Governing Sustainable Tourism Mobility: Psychological and Behavioural Approaches (vol. 43). Routledge.

[18] COHEN, S. A.; HIGHAM, J. E. y REIS, A. C. (2013). «Sociological barriers to developing sustainable discretionary air travel behaviour». Journal of Sustainable Tourism, 21 (7), 982-998. 
[19] DANN, G. M. (1981). «Tourist motivation an appraisal». Annals of Tourism Research, $8(2), 187-219$.

[20] DEL BOSQUE, I. R. y SAN MARTÍN, H. (2008). «Tourist satisfaction a cognitiveaffective model». Annals of Tourism Research, 35 (2), 551-573.

[21] DIMANCHE, F. (2003). «The Louisiana tax-free shopping program for international visitors: A case study». Journal of Travel Research, 41, 311-314.

[22] DOLNICAR, S.; KNEZEVIC CVELBAR, L. y GRÜN, B. (2017). «Do proenvironmental appeals trigger pro-environmental behavior in hotel guests?». Journal of Travel Research, 56 (8), 988-997.

[23] DORAN, R.; HANSS, D. y LARSEN, S. (2017). «Intentions to make sustainable tourism choices: do value orientations, time perspective, and efficacy beliefs explain individual differences?». Scandinavian Journal of Hospitality and Tourism, 17 (3), 223 238.

[24] FONT, X.; WALMSleY, A.; COGOTTI, S.; McCOMBES, L. y HÄUSLER, N. (2012). «Corporate social responsibility: The disclosure-performance gap». Tourism Management, 33 (6), 1544-1553.

[25] GIFFORD, R. y NILSSON, A. (2014). «Personal and social factors that influence proenvironmental concern and behaviour: A review». International Journal of Psychology, 49 (3), 141-157.

[26] GRETZEL, U.; FESENMAIER, D. R. y O’LEARY, J. T. (2006). «The transformation of consumer behaviour». Tourism Business Frontiers, 9-18.

[27] GNOTH, J. (1997). «Tourism motivation and expectation formation». Annals of Tourism Research, 24 (2), 283-304.

[28] GOLDSTEIN, N. J.; CIALDINI, R. B. y GRISKEVICIUS, V. (2008). «A room with a viewpoint: Using social norms to motivate environmental conservation in hotels». Journal of Consumer Research, 35, 472-482.

[29] GOLDSTEIN, N. J.; GRISKEVICIUS, V. y CIALDINI, R. B. (2011). «Reciprocity by proxy: A novel influence strategy for stimulating cooperation». Administrative Science Quarterly, 56, 411-473.

[30] GOOROOCHURN, N. y MILNER, C. (2005). «Assessing indirect tax reform in a tourism-dependant developing country». World Development, 33 (7), 1183-1200.

[31] GÖSSLING, S.; BRODERICK, J.; UPHAM, P.; CERON, J.P.; DUBOIS, G.; PEETERS, P. y STRASDAS, W. (2007). «Voluntary carbon offsetting schemes for aviation: Efficiency, credibility and sustainable tourism». Journal of Sustainable Tourism, 15 (3), 223-248.

[32] GÖSSLING, S. (2015). «Low-carbon and post-carbon travel and destinations». The Routledge Handbook of Tourism and Sustainability, 472.

[33] GÖSSLING, S. y BUCKLEY, R. (2016). «Carbon labels in tourism: persuasive communication?». Journal of Cleaner Production, 111, 358-369.

[34] GÖSSLING, S. N. y ARNELL, N. W. (2016). «A global assessment of the impact of climate change on water scarcity». Climatic Change, 134 (3), 371-385.

[34] GÖSSLING, S.; SCOTT, D.; HALL, C. M.; CERON, J. P. y DUBOIS, G. (2012). «Consumer behaviour and demand response of tourists to climate change». Annals of Tourism Research, 39 (1), 36-?.

[35] GOLDSTEIN, N. J.; GRISKEVICIUS, V. y CIALDINI, R. B. (2011). «Reciprocity by proxy: A novel influence strategy for stimulating cooperation». Administrative Science Quarterly, 56, 411-473. 
[36] GRETZEL, U.; FESENMAIER, D. R.; FORMICA, S. y O’LEARY, J. T. (2006). «Searching for the future: Challenges faced by destination marketing organizations». Journal of Travel Research, 45 (2), 116-126.

[37] HALL, C. M. (2013). «Framing behavioural approaches to understanding and governing sustainable tourism consumption: beyond neoliberalism, 'nudging' and 'green growth'?». Journal of Sustainable Tourism, 21, 1091-1109.

[38] HALL, C. M. (2016). «Intervening in academic interventions: framing social marketing's potential for successful sustainable tourism behavioural change». Journal of Sustainable Tourism, 24 (3), 350-375.

[39] HAN, H. (2015). «Travelers' pro-environmental behavior in a green lodging context: Converging value-belief-norm theory and the theory of planned behavior». Tourism Management, 47, 164-177.

[40] HARES, A.; DICKINSON, J. y WILKES, K. (2010). «Climate change and the air travel decisions of UK tourists». Journal of Transport Geography, 18 (3), 466-473.

[41] HASSAN, L. M.; SHIU, E. y SHAW, D. (2016). "Who says there is an intentionbehaviour gap? Assessing the empirical evidence of an intention-behaviour gap in ethical consumption». Journal of Business Ethics, 136 (2), 219-236.

[42] HEDLUND, T. (2011). «The impact of values, environmental concern, and willingness to accept economic sacrifices to protect the environment on tourists' intentions to buy ecologically sustainable tourism alternatives». Tourism and Hospitality Research, 11 (4), 278-288.

[43] HIBBERT, J. F.; DICKINSON, J. E.; GÖSSLING, S. y CURTIN, S. (2013). «Identity and tourism mobility: an exploration of the attitude-behaviour gap». Journal of Sustainable Tourism, 21 (7), 999-1016.

[44] HIGHAM, J. E. y COHEN, S. A. (2011). «Canary in the coalmine: Norwegian attitudes towards climate change and extreme long-haul air travel to Aotearoa/New Zealand». Tourism Management, 32 (1), 98-105.

[45] HIGHAM, J.; REIS, A. y COHEN, S. A. (2016). «Australian climate concern and the 'attitude-behaviour gap». Current Issues in Tourism, 19 (4), 338-354.

[46] HOPPE, E. I. y KUSTERER, D. J. (2011). «Behavioral biases and cognitive reflection». Economics Letters, 110 (2), 97-100.

[47] HSU, C. H. y HUANG, S. (2012). «An extension of the theory of planned behavior model for tourists». Journal of Hospitality \& Tourism Research, 36 (3), 390-417.

[48] INMAN, J. J.; PETER, A. C. y RAGHUBIR, P. (1997). «Framing the deal: The role of restrictions in accentuating deal value». Journal of Consumer Research, 24 (1), 68-79.

[49] IMRAN, S.; ALAM, K. y BEAUMONT, N. (2014). «Environmental orientations and environmental behaviour: Perceptions of protected area tourism stakeholders». Tourism management, 40, 290-299.

[50] JAMAL, T. y CAMARGO, B. A. (2014). «Sustainable tourism, justice and an ethic of care: Toward the just destination». Journal of Sustainable Tourism, 22 (1), 11-30.

[51] JUVAN, E. y DOLNICAR, S. (2014). «The Attitude-Behaviour Gap in Sustainable Tourism». Annals of Tourism Research, 48, 76-95.

[52] JUVAN, E.; GRÜN, B. y DOLNICAR, S. (2017). «Biting Off More Than They Can Chew: Food Waste at Hotel Breakfast Buffets». Journal of Travel Research, 0047287516688321. 
[53] JUVAN, E.; RING, A.; LEISCH, F. y DOLNICAR, S. (2016). «Tourist segments' justifications for behaving in an environmentally unsustainable way». Journal of Sustainable Tourism, 24 (11), 1506-1522.

[54] KARLSSON, L. y DOLNICAR, S. (2016). «Does eco certification sell tourism services? Evidence from a quasi-experimental observation study in Iceland». Journal of Sustainable Tourism, 24 (5), 694-714.

[55] KING, J. (2002). «Destination Marketing Organisations: Connecting the Experience Rather than Promoting the Place». Journal of Vacation Marketing, 8 (2), 105-108.

[56] LEE, C. K.; MJELDE, J. W.; KIM, T. K. y LEE, H. M. (2014). «Estimating the intentionbehavior gap associated with a mega event: The case of the Expo 2012 Yeosu Korea». Tourism Management, 41, 168-177.

[57] LERNER, J. S. y KELTNER, D. (2000). «Beyond Valence: Toward a model of emotionspecific influences on judgment and choice». Cognition and Emotion, 14 (4), 473-493.

[58] LUCAS, K.; BROOKS, M.; DAMTON, A. y JONES, J. E. (2008). «Promoting proenvironmental behaviour: existing evidence and policy implications». Environmental Science \& Policy, 11.5, 456-466.

[59] MAIR, J. y BERGIN-SEERS, S. (2010). «The Effect of Interventions on the Environmental Behaviour of Australian Motel Guests». Tourism and Hospitality Research, 10 (4), 255-268.

[60] MAC CANNELL, D. (2013). The tourist: A new theory of the leisure class. Univ. of California Press.

[61] MIHALIC, T. (2016). «Sustainable-responsible tourism discourse-Towards 'responsustable' tourism». Journal of Cleaner Production, 111, 461-470.

[62] MILLER, D.; MERRILEES, B. y COGHLAN, A. (2015). «Sustainable urban tourism: understanding and developing visitor pro-environmental behaviours». Journal of Sustainable Tourism, 23 (1), 26-46.

[63] MOSER, S.C. (2014). «Communicating adaptation to climate change: the art and science of public engagement when climate change comes home». Wiley Interdisciplinary Reviews: Climate Change, 5 (3), 337-358.

[64] OECHSSLER, J.; ROIDER, A. y SCHMITZ, P. W. (2009). «Cognitive abilities and behavioral biases». Journal of Economic Behavior \& Organization, 72 (1), 147-152.

[65] O'NEILL, M. y PALMER, A. (2004). «Cognitive dissonance and the stability of service quality perceptions». Journal of Services Marketing, 18 (6), 433-449.

[66] PALMER, T. y RIERA, A. (2003). «Tourism and environmental taxes. With special references to the Balearic ecotax». Tourism Management, 24, 665-674.

[67] PALMER-TOUS, T.; RIERA-FONT, A. y ROSSELLÓ-NADAL, J. (2007). «Taxing tourism: The case of rental cars in Mallorca». Tourism Management, 28, 271-279.

[68] SCOTT, D.; GÖSSLING, S. y HALL, C. M. (2012b). «International tourism and climate change». Wiley Interdisciplinary Reviews: Climate Change, 3 (3), 213-232.

[69] SCOTT, D.; GÖSSLING, S.; HALL, C. M. y PEETERS, P. (2016). «Can tourism be part of the decarbonized global economy? The costs and risks of alternate carbon reduction policy pathways». Journal of Sustainable Tourism, 24 (1), 52-72.

[70] SHANG, J.; BASIL, D. Z. y WYMER, W. (2010). «Using social marketing to enhance hotel resuse programs». Journal of Business Research, 63 (2), 166-172.

[71] SHARPLEY, R. (2000). «Tourism and sustainable development: Exploring the theoretical divide». Journal of Sustainable tourism, 8 (1), 1-19. 
[72] SHENG, L. y TSUI, Y. (2009). «Taxing tourism: enhancing or reducing welfare?». Journal of Sustainable Tourism, 17 (5), 627-635.

[73] SIRAKAYA, E. y WOODSIDE, A. G. (2005). «Building and testing theories of decision making by travellers». Tourism Management, 26 (6), 815-832.

[74] STEG, L. y VLEK, C. (2009). «Encouraging pro-environmental behaviour: An integrative review and research agenda». Journal of Environmental Psychology, 29 (3), 309-317.

[75] STERN, P. (2000). «Toward a Coherent Theory of Environmentally Significant Behaviour». Journal of Social Issues, 56 (3), 407-24.

[80] TANFORD, S. y MONTGOMERY, R. (2015). «The effects of social influence and cognitive dissonance on travel purchase decisions». Journal of Travel Research, 54 (5), 596-610.

[81] TENG, Y. M.; WU, K. S. y LIU, H. H. (2015). «Integrating altruism and the theory of planned behavior to predict patronage intention of a green hotel». Journal of Hospitality \& Tourism Research, 39 (3), 299-315.

[82] TUAN, L. T. (2017). «Activating tourists' citizenship behavior for the environment: the roles of CSR and frontline employees' citizenship behavior for the environment». Journal of Sustainable Tourism, 1-26.

[83] TUFAN, E.; AYCAN, M.; IMER, Y.; HAMARAT, B. y DURAN, E. (2016). «Does Human Stick on Reference Price (Anchoring Bias)? A Social Experiment on Tourism Sector». International Journal of Economics \& Business Administration (IJEBA), 4 (2), 97-106.

[84] VAN DER LINDEN, S. (2015). «Intrinsic Motivation and Pro-environmental Behaviour». Nature Climate Change, 5 (7), 612-13.

[85] VERMEIR, I. y VAN KENHOVE, P. (2005). «The influence of need for closure and perceived time pressure on search effort for price and promotional information in a grocery shopping context». Psychology \& Marketing, 22 (1), 71-95.

[86] VÖRÖSMARTY, C. J.; GREEN, P.; SALISBURY, J. y LAMMERS, R. B. (2000). «Global water resources: vulnerability from climate change and population growth». Science, 289 (5477), 284-288.

[87] VU, H. N.; HALES, R.; ARCODIA, C. y ANDRADE, L. B. D. (2015). «A peoplecentred approach to destination planning and management: A paradigm shift toward sustainable development». CAUTHE 2015: Rising Tides and Sea Changes: Adaptation and Innovation in Tourism and Hospitality, 745.

[88] WALIGO, V. M.; CLARKE, J. y HAWKINS, R. (2013). «Implementing sustainable tourism: A multi-stakeholder involvement management framework». Tourism Management, 36, 342-353. 